PROCEEDINGS OF THE

AMERICAN MATHEMATICAL SOCIETY

Volume 130, Number 12, Pages 3651-3660

S 0002-9939(02)06580-2

Article electronically published on May 1, 2002

\title{
ESTIMATES FOR VISCOSITY SOLUTIONS OF PARABOLIC EQUATIONS WITH DIRICHLET BOUNDARY CONDITIONS
}

\author{
G. GRIPENBERG
}

(Communicated by David S. Tartakoff)

\begin{abstract}
It is shown how one can get upper bounds for $|u-v|$ when $u$ and $v$ are the (viscosity) solutions of

$$
u_{t}-\alpha\left(D_{x} u\right) \Delta_{x} u=0 \text { and } v_{t}-\beta\left(D_{x} v\right) \Delta_{x} v=0,
$$

respectively, in $(0, \infty) \times \Omega$ with Dirichlet boundary conditions. Similar results are obtained for some other parabolic equations as well, including certain equations in divergence form.
\end{abstract}

\section{INTRODUCTION}

In this paper we study the problem of how to estimate the difference between the (viscosity) solutions $u$ and $v$ of the parabolic equations

$$
u_{t}-\alpha\left(D_{x} u\right) \Delta_{x} u=0 \quad \text { and } \quad v_{t}-\beta\left(D_{x} v\right) \Delta_{x} v=0,
$$

respectively, in $(0, T) \times \Omega$ with Dirichlet boundary conditions when $u(0, \underline{x})$ and $v(0, \underline{x})$ are given and $\Omega$ is an open and convex subset of $\mathbb{R}^{d}$. Here both $\alpha$ and $\beta$ are nonnegative continuous functions and if for example $u(0, \underline{x})=v(0, \underline{x})$ is Hölder continuous with exponent $\theta$, then we can conclude that $|u(t, x)-v(t, x)| \leq$ $C t^{\frac{\theta}{2}} \sup _{\mathbf{q} \in \mathbb{R}^{d}}|\sqrt{\alpha(\mathbf{q})}-\sqrt{\beta(\mathbf{q})}|^{\theta}$ for all $t \geq 0$ and $x \in \Omega$.

For a general introduction to, and definitions of, viscosity solutions, see for example [2] or [3] and the references mentioned there.

Instead of equation (1) we can study equations of the form

$$
u_{t}-\operatorname{div}\left(a\left(\left|D_{x} u\right|\right) D_{x} u\right)=0 \quad \text { and } \quad v_{t}-\operatorname{div}\left(b\left(\left|D_{x} u\right|\right) D_{x} u\right)=0 .
$$

These results can be combined to results for equations of the form

$$
\begin{aligned}
& u_{t}+f\left(u, D_{x} u, D_{x}^{2} u\right)-\operatorname{div}\left(a\left(\left|D_{x} u\right|\right) D_{x} u\right)-\alpha\left(D_{x} u\right) \Delta_{x} u=0, \text { in }(0, \infty) \times \Omega, \\
& u(t, x)=0, \quad t>0, \quad x \in \partial \Omega \\
& u(0, x)=u_{0}(x), \quad x \in \Omega
\end{aligned}
$$

Received by the editors July 23, 2001.

2000 Mathematics Subject Classification. Primary 35K55, 35K65, 35K20.

Key words and phrases. Viscosity solution, parabolic, dependence on data, Dirichlet boundary condition. 
and

$$
\begin{aligned}
& v_{t}+g\left(v, D_{x} v, D_{x}^{2} v\right)-\operatorname{div}\left(b\left(\left|D_{x} u\right|\right) D_{x} u\right)-\beta\left(D_{x} v\right) \Delta_{x} v=0, \text { in }(0, \infty) \times \Omega, \\
& v(t, x)=0, \quad t>0, \quad x \in \partial \Omega, \\
& v(0, x)=v_{0}(x), \quad x \in \Omega .
\end{aligned}
$$

Here $D_{x} u$ denotes the (spatial) gradient of $u$ and $D_{x}^{2} u$ denotes the $d \times d$ matrix of second derivatives. The trace of this matrix is the Laplacian $\Delta_{x} u$. The reason for writing the equations in this special form is that one gets somewhat better estimates for terms of the form $-\operatorname{div}\left(a\left(\left|D_{x} u\right|\right) D_{x} u\right)=-a\left(\left|D_{x} u\right|\right) \Delta_{x} u-\frac{a^{\prime}\left(\mid D_{x} u\right) \mid}{\left|D_{x} u\right|}\left\langle D_{x} u, D_{x}^{2} u D_{x} u\right\rangle$ and $-\alpha\left(D_{x} u\right) \Delta_{x} u$ than for the more general case $f\left(u, D_{x} u, D_{x}^{2} u\right)$. (Here $\langle\cdot, \cdot\rangle$ denotes the standard inner product in $\mathbb{R}^{d}$.) The fundamental monotonicity condition is that $f$ and $g$ are nondecreasing in their first and nonincreasing in their third variable, and similarly that $a$ and $b$ are nonincreasing and that $a+\alpha$ and $b+\beta$ are nonnegative functions.

Observe that we assume that the domain $\Omega$ is convex, but we do not have to assume that it is bounded. The boundary condition at points at infinity are here taken to be that the solution is bounded, but it would be sufficient to have a restriction on the growth rate at infinity.

In [1] the problem considered above was studied in the case of Neumann boundary conditions (and with $a=b=0$ ) and here we shall consider the case of Dirichlet conditions imposed at the boundary points. It turns out that at least if one makes the additional assumptions that $f(0, \mathbf{q}, 0)=0$ and that $g(0, \mathbf{q}, 0)=0$ for all $\mathbf{q} \in \mathbb{R}^{d}$, then one can fairly easily obtain the same conclusion as in [1]. Another difference compared to [1] is that here we state for completeness a result concerning existence as well.

\section{Statement of Results}

We let $\mathcal{S}(d)$ be the space of symmetric real $d \times d$ matrices with order defined by $A \geq B$ if $\langle\mathbf{p}, A \mathbf{p}\rangle \geq\langle\mathbf{p}, B \mathbf{p}\rangle$ for every $\mathbf{p} \in \mathbb{R}^{d}$. Furthermore, $\mathcal{C}, \mathcal{C}^{1}$, and $\mathcal{B U C}$ stand for continuous, continuously differentiable, and bounded and uniformly continuous, respectively.

Theorem 1. Assume that $d \geq 1$, and that

(i) $\Omega \subset \mathbb{R}^{d}$ is an open and convex set;

(ii) $f \in \mathcal{C}\left(\mathbb{R} \times \mathbb{R}^{d} \times \mathcal{S}(d) ; \mathbb{R}\right)$ and $g \in \mathcal{C}\left(\mathbb{R} \times \mathbb{R}^{d} \times \mathcal{S}(d) ; \mathbb{R}\right)$, and both functions are nondecreasing in their first and nonincreasing in their third argument;

(iii) $f(0, \mathbf{q}, 0)=g(0, \mathbf{q}, 0)=0$ for all $\mathbf{q} \in \mathbb{R}^{d}$;

(iv) $\alpha$ and $\beta \in \mathcal{C}\left(\mathbb{R}^{d} ; \mathbb{R}\right)$, $a$ and $b \in \mathcal{C}\left(\mathbb{R}^{+} ; \mathbb{R}\right) \cap \mathcal{C}^{1}((0, \infty) ; \mathbb{R})$ with $\lim _{r \downarrow 0} r a^{\prime}(r)=$ $\lim _{r \downarrow 0} r b^{\prime}(r)=0$. Moreover, $\alpha(\mathbf{q})+a(|\mathbf{q}|) \geq 0, \beta(\mathbf{q})+b(|\mathbf{q}|) \geq 0, a^{\prime}(r) \geq 0$ and $b^{\prime}(r) \geq 0$ for all $\mathbf{q} \in \mathbb{R}^{d}$ and $r>0$;

(v) $u_{0}$ and $v_{0} \in \mathcal{B U C}(\bar{\Omega} ; \mathbb{R})$ with $u_{0}(x)=v_{0}(x)=0$ for $x \in \partial \Omega$. Moreover, $\omega: \mathbb{R}^{+} \rightarrow \mathbb{R}^{+}$is a concave, nondecreasing, and continuous function with $\omega(0)=0$ such that

$$
\begin{aligned}
\left|u_{0}(x)-u_{0}(y)\right| & \leq \omega(|x-y|), \\
\left|v_{0}(x)-v_{0}(y)\right| & \leq \omega(|x-y|), \quad x, y \in \Omega .
\end{aligned}
$$


Then there are unique bounded solutions $u$ and $v$ of equations (2) and (3), respectively, and for each $t \geq 0, x \in \bar{\Omega}$, and $\lambda>0$, we have

$$
\begin{aligned}
& |u(t, x)-v(t, x)| \leq \sup _{y \in \bar{\Omega}}\left|u_{0}(y)-v_{0}(y)\right|+\sup _{r \geq 0}\left(\omega(r)-\frac{\lambda}{2} r^{2}\right) \\
& +t \sup _{(r, \mathbf{q}, X) \in D_{\lambda}}\left(|g(r, \mathbf{q}, X)-f(r, \mathbf{q}, X)|+3 \lambda\left(\sqrt{|\mathbf{q}| a^{\prime}(|\mathbf{q}|)}-\sqrt{|\mathbf{q}| b^{\prime}(|\mathbf{q}|)}\right)^{2}\right. \\
& \left.+3 \lambda d(\sqrt{a(|\mathbf{q}|)+\alpha(\mathbf{q})}-\sqrt{b(|\mathbf{q}|)+\beta(\mathbf{q})})^{2}\right),
\end{aligned}
$$

where

$$
\begin{aligned}
& D_{\lambda} \stackrel{\text { def }}{=}\left(\mathcal{R}\left(u_{0}\right)\right.\left.\cup \mathcal{R}\left(v_{0}\right)\right) \\
& \times\left\{\mathbf{q} \in \mathbb{R}^{d}|| \mathbf{q} \mid \leq \sqrt{2 \lambda} \sqrt{\operatorname{diam}\left(\mathcal{R}\left(u_{0}\right) \cup \mathcal{R}\left(v_{0}\right)\right)}\right\} \\
& \quad \times\{X \in \mathcal{S}(d) \mid\|X\| \leq 3 \lambda\} .
\end{aligned}
$$

Here $\mathcal{R}\left(u_{0}\right)$ denotes the range $\left\{u_{0}(x) \mid x \in \bar{\Omega}\right\}$ of $u_{0}$. Recall also that if $\omega$ is Hölder continuous, that is, $\omega(r) \leq C r^{\theta}$ for $r \geq 0$, where $\theta \in(0,1]$, then $\sup _{r \geq 0}\left(\omega(r)-\frac{\lambda}{2} r^{2}\right) \leq \frac{1}{2} C^{\frac{2}{2-\theta}} \lambda^{-\frac{\theta}{2-\theta}}$ when $\lambda>0$.

Theorem 1 states that the solution is unique among all bounded solutions. This result can certainly be improved but some restrictions on the solutions are needed because it is a well known fact that, e.g., the heat equation has null solutions when $\Omega=\mathbb{R}$, i.e., solutions that are zero at $t=0$ but nonzero for $t>0$.

Clearly, one should in Theorem 1 choose $\lambda$ so that the right hand side of (5) is as small as possible. In general this is quite difficult, but we state a simple result that one gets when $f=g$ and $a=b=0$.

Corollary 2. Let the assumptions of Theorem 1 hold with $f=g, a=b=0$, and $\omega(\underline{r})=C \underline{r}^{\theta}$, for some $C \geq 0$ and $\theta \in(0,1]$. Then for each $t \geq 0$ and $x \in \bar{\Omega}$ we have

$$
\begin{aligned}
|u(t, x)-v(t, x)| & \leq \sup _{y \in \bar{\Omega}}\left|u_{0}(y)-v_{0}(y)\right| \\
& +\sqrt{6} C(t d)^{\frac{\theta}{2}} \sup _{\mathbf{q} \in \mathbb{R}^{d}}|\sqrt{\alpha(\mathbf{q})}-\sqrt{\beta(\mathbf{q})}|^{\theta} .
\end{aligned}
$$

In order to see how good this estimate really is, consider the case where $d=1$, $\Omega=\mathbb{R}, f=g=\alpha=0, \beta(\underline{\mathbf{q}})=c>0$, and where $u(0, \underline{x})=v(0, \underline{x})=\left(1-|\underline{x}|^{\theta}\right)^{+}$for some $\theta \in(0,1]$ where $(\underline{a})^{+}=\max \{\underline{a}, 0\}$. Then a calculation shows that

$$
u(t, 0)-v(t, 0) \geq(c t)^{\frac{\theta}{2}} \frac{2^{1+\theta}}{\sqrt{\pi}} \int_{0}^{\frac{1}{2 \sqrt{c t}}} s^{\theta} \mathrm{e}^{-s^{2}} \mathrm{~d} s, \quad t \geq 0
$$

whereas Corollary 2 says that

$$
u(t, 0)-v(t, 0) \leq(c t)^{\frac{\theta}{2}} \sqrt{6}, \quad t \geq 0 .
$$

Next we state an application of Theorem 1 to the question of how the solution of the equation $u_{t}-\operatorname{div}\left(\left|D_{x} u\right|^{p-2} D_{x} u\right)=0$ depends on the parameter $p>2$.

Corollary 3. Let the assumptions of Theorem [1] hold with $f=g, \alpha=\beta=0$, $\omega(\underline{r})=C \underline{r}^{\theta}$, for some $C>0$ and $\theta \in(0,1]$, and $a(\underline{r})=\underline{r}^{p-2}$ and $b(\underline{r})=\underline{r}^{q-2}$. 
Then for each $\epsilon>0$ there is a constant $K$ depending on $\mathcal{R}\left(u_{0}\right) \cup \mathcal{R}\left(v_{0}\right)$, $\epsilon$, and on $C$ such that for every $t \geq 0$ and $x \in \bar{\Omega}$

$$
|u(t, x)-v(t, x)| \leq \sup _{x \in \bar{\Omega}}\left|u_{0}(x)-v_{0}(x)\right|+K(\sqrt{p d t}(p-q))^{\frac{4 \theta}{2 \theta+p(2-\theta)+\epsilon}},
$$

provided $p \geq q \geq 2+\epsilon$ and $\sqrt{p d t}(p-q) \leq 1$.

\section{Proof of Theorem 1}

We use the notation $F(\underline{r}, \underline{\mathbf{q}}, \underline{X})=f(\underline{r}, \underline{\mathbf{q}}, \underline{X})-\frac{a^{\prime}(|\underline{\mathbf{q}}|)}{|\underline{\mathbf{q}}|}\langle\underline{\mathbf{q}}, X \underline{\mathbf{q}}\rangle-(a(\underline{\mathbf{q}})+\alpha(\underline{\mathbf{q}})) \operatorname{tr}(\underline{X})$ and $G(\underline{r}, \underline{\mathbf{q}}, \underline{X})=g(\underline{r}, \underline{\mathbf{q}}, \underline{X})-\frac{b^{\prime}(|\mathbf{q}|)}{|\underline{\mathbf{q}}|}\langle\underline{\mathbf{q}}, X \underline{\mathbf{q}}\rangle-(b(\underline{\mathbf{q}})+\beta(\underline{\mathbf{q}})) \operatorname{tr}(\underline{X})$ where $\operatorname{tr}(X)$ denotes the trace of $X$ and where $\frac{a^{\prime}(|\mathbf{0}|)}{|\mathbf{0}|}\langle\mathbf{0}, X \mathbf{0}\rangle=\frac{b^{\prime}(|\mathbf{0}|)}{|\mathbf{0}|}\langle\mathbf{0}, X \mathbf{0}\rangle=0$.

Assume that $u$ is an upper semi-continuous function on $\mathbb{R}^{+} \times \bar{\Omega}$ such that $\sup _{t \in[0, T], x \in \bar{\Omega}} u(t, x)<\infty$ for all $T \in(0, \infty)$ and $u$ is a subsolution of (2) (so that $u(t, x) \leq 0$ when $t \geq 0$ and $x \in \partial \Omega$ and $u(0, x) \leq u_{0}(x)$ when $\left.x \in \bar{\Omega}\right)$. Correspondingly, we assume that $v$ is a lower semi-continuous function on $\mathbb{R}^{+} \times \bar{\Omega}$ such that inf $\operatorname{in}_{t \in, T], x \in \bar{\Omega}} v(t, x)>-\infty$ for all $T \in(0, \infty)$ and $v$ is a supersolution of (3).

We shall later need the fact that comparison holds for equation (2) (and then also for (3) ). This is the same as having $u(t, x) \leq v(t, x)$ for all $t \geq 0$ and all $x \in \bar{\Omega}$ provided $f=g, \alpha=\beta, a=b$, and $u_{0} \leq v_{0}$. This result is proven in [3] Thm 8.3] in the case where $\Omega$ is bounded. It is not clear that this proof easily extends to the case where $\Omega$ is unbounded but has a boundary, so we have first to establish a result on the behaviour of solutions close to the boundary.

Let $\widehat{u_{0}}$ be the concave envelope of $u_{0}(\underline{x})$ (i.e., the infimum of all affine functions that are greater than $u_{0}$ on $\left.\bar{\Omega}\right)$. Since $u_{0}(x)=0$ when $x \in \partial \Omega$ we have $\widehat{u_{0}}(\underline{x}) \geq 0$ and it follows from (iii) that the function $(t, x) \mapsto \widehat{u_{0}}(x)$ is a supersolution of (2). If comparison holds for (2), then we would have

$$
u(t, x) \leq \widehat{u_{0}}(x), \quad t \geq 0, \quad x \in \bar{\Omega}
$$

Next we give a direct proof of this fact, which is later used to prove that comparison in fact holds. A similar argument shows that

$$
v(t, x) \geq \breve{v_{0}}(x), \quad t \geq 0, \quad x \in \bar{\Omega},
$$

where $\widetilde{v_{0}}$ is the convex envelope of $v_{0}$.

Let $\mu$ be an arbitrary affine function such that

$$
u_{0}(x) \leq \mu(x), \quad x \in \bar{\Omega} .
$$

Let $\epsilon>0, T>0, r \geq 0$, and let

$$
U_{r}(t, x)=r+r t+\mu(x)+\epsilon|x|^{2}+\frac{\epsilon}{T-t}, \quad 0 \leq t<T, \quad x \in \bar{\Omega} .
$$

Since $u$ is upper semi-continuous and bounded from above there are by (9) positive numbers $r$ such that $u(t, x) \leq U_{r}(t, x)$ for all $t \in[0, T)$ and $x \in \bar{\Omega}$. Let $r_{0}$ be the infimum of these numbers. Thus we have

$$
u(t, x) \leq r_{0}+r_{0} t+\mu(x)+\epsilon|x|^{2}+\frac{\epsilon}{T-t}, \quad 0 \leq t<T, \quad x \in \bar{\Omega} .
$$


If $r_{0}>0$, then there must be some point $\left(t_{0}, x_{0}\right)$ where we have equality (otherwise we could make $r_{0}$ smaller), that is,

$$
u\left(t_{0}, x_{0}\right)=U_{r_{0}}\left(t_{0}, x_{0}\right) .
$$

(Note that $r_{0}, t_{0}$, and $x_{0}$ depend on $\epsilon$.) By the definition of $U_{r}$ we know that $u\left(t_{0}, x_{0}\right)>0$ and therefore we must have $t_{0}>0$ and $x_{0} \in \Omega$. Since $u$ is a subsolution it follows that

$$
r_{0}+\frac{\epsilon}{\left(T-t_{0}\right)^{2}}+F\left(u\left(t_{0}, x_{0}\right), D_{x} \mu\left(x_{0}\right)+2 \epsilon x_{0}, D_{x}^{2} \mu\left(x_{0}\right)+2 \epsilon I\right) \leq 0 .
$$

Because $\mu$ is affine we have $D_{x}^{2} \mu\left(x_{0}\right)=0$ so that we see from assumption (iii) that

$$
r_{0}+F\left(0, D_{x} \mu\left(x_{0}\right)+2 \epsilon x_{0}, 2 \epsilon I\right) \leq 0 .
$$

Now $r_{0}$ and $x_{0}$ depend on $\epsilon$ and since $\epsilon\left|x_{0}\right|^{2}$ must be bounded by the supremum of $u(\underline{t}, \underline{x})$ on $[0, T] \times \bar{\Omega}$, we conclude that $\lim _{\epsilon \downarrow 0} \epsilon\left|x_{0}\right|=0$. Using assumption (iii) and the fact that $D_{x} \mu$ is constant we conclude that $\lim \sup _{\epsilon \downarrow 0} r_{0}=0$. Since $\epsilon$ was arbitrary, it follows from (10) that $u(t, x) \leq \mu(x)$ when $0 \leq t<T$ and $x \in \bar{\Omega}$. But since $T$ and $\mu$ were arbitrary, and since $\widehat{u}_{0}$ is the infimum of all affine functions satisfying (9), we conclude that we actually have (7).

Next we shall prove that

$$
\widehat{u_{0}}(x) \leq \omega(|x-y|), \quad x \in \Omega, \quad y \in \partial \Omega .
$$

(We could easily prove that $\omega$ is a modulus of continuity for $\widehat{u_{0}}$ in $\bar{\Omega}$.) To prove this inequality, suppose that $x \in \Omega$ and $y \in \partial \Omega$ and let $\epsilon>0$ be arbitrary. By [5, Corollary 17.1.5, p. 157] there are points $x_{0}, x_{1}, \ldots, x_{d} \in \bar{\Omega}$ and nonnegative numbers $\lambda_{0}, \lambda_{1}, \ldots, \lambda_{d}$ such that $\sum_{j=0}^{d} \lambda_{j}=1, \sum_{j=0}^{d} \lambda_{j} x_{j}=x$ and

$$
\widehat{u_{0}}(x) \leq \sum_{j=0}^{d} \lambda_{j} u_{0}\left(x_{j}\right)+\epsilon .
$$

Suppose there are numbers $c_{j} \geq 0$ such that $x_{j}+c_{j}(y-x) \in \bar{\Omega}, j=0,1, \ldots, d$, and such that $\beta \stackrel{\text { def }}{=} \sum_{j=0}^{d} \lambda_{j} c_{j}>1$. Then $\sum_{j=0}^{d} \lambda_{j}\left(x_{j}+c_{j}(y-x)\right)=x+\beta(y-x) \in \bar{\Omega}$, but this implies, since $\Omega$ is convex and open, $x \in \Omega$, and $\beta>1$ that $y \notin \partial \Omega$ which is a contradiction. Thus we can for all $j=0,1, \ldots, d$ find numbers $c_{j} \geq 0$ such that $x_{j}+c_{j}(y-x) \in \partial \Omega$ if $\lambda_{j}>0$, and such that $\beta=\sum_{j=0}^{d} \lambda_{j} c_{j} \leq 1$.

By (घ) we conclude that if $x_{j}+c_{j}(y-x) \in \partial \Omega$, then $u_{0}\left(x_{j}\right) \leq \omega\left(c_{j}|y-x|\right)$, and therefore it follows from (12) and from the facts that $\beta \leq 1$ and $\omega$ is nondecreasing and concave that

$$
\begin{aligned}
\widehat{u_{0}}(x) & \leq \sum_{j=0}^{d} \lambda_{j} u_{0}\left(x_{j}\right)+\epsilon \leq \sum_{j=0}^{d} \lambda_{j} \omega\left(c_{j}|y-x|\right)+\epsilon \\
& \leq \omega\left(\sum_{j=0}^{d} \lambda_{j} c_{j}|y-x|\right)+\epsilon=\omega(\beta|x-y|)+\epsilon \leq \omega(|x-y|)+\epsilon .
\end{aligned}
$$

Since $\epsilon>0$ was arbitrary, this gives the inequality in (11).

Combining (7) with (11) we conclude that

$$
u(t, x) \leq \omega(|x-y|), \quad x \in \bar{\Omega}, \quad y \in \partial \Omega .
$$


Similarly we also get, using the convex envelope of $v_{0}$ instead, that

$$
v(t, x) \geq-\omega(|x-y|), \quad x \in \bar{\Omega}, \quad y \in \partial \Omega .
$$

Now we proceed using the same techniques as in [1]. Define

$$
E_{0} \stackrel{\text { def }}{=} \sup _{x \in \bar{\Omega}}\left(u_{0}(x)-v_{0}(x)\right)^{+} \quad \text { and } \quad M_{0}=\operatorname{diam}\left(\mathcal{R}\left(u_{0}\right) \cup \mathcal{R}\left(v_{0}\right)\right) .
$$

Let $T>0$ and $\epsilon>0$ be arbitrary and let

$$
\sigma \stackrel{\text { def }}{=} \sup _{\substack{t \in[0, T) \\ x \in \Omega}}\left(u(t, x)-v(t, x)-\frac{\epsilon}{T-t}-\frac{\epsilon}{2}|x|^{2}\right)-E_{0} .
$$

If $\sigma \leq 0$ for arbitrarily small $\epsilon$ we get directly the desired conclusion, so we shall assume that $\sigma>0$. Let $\delta \in(0,1)$ and $\lambda \geq \epsilon$ be arbitrary and define

$$
\begin{aligned}
\psi(t, x, y) \stackrel{\text { def }}{=} u(t, x)-v(t, y) & -\frac{\epsilon}{T-t}-\frac{(1-\delta) \sigma}{T} t \\
& -\frac{1}{2} \lambda|x-y|^{2}-\frac{1}{2} \epsilon|x|^{2}, \quad t \in[0, T), \quad x, y \in \bar{\Omega}
\end{aligned}
$$

First we observe, by considering the possibility that $x=y$, that we must have

$$
\sup _{\substack{t \in[0, T) \\ x, y \in \Omega}} \psi(t, x, y) \geq \sigma+E_{0}-(1-\delta) \sigma=\delta \sigma+E_{0} .
$$

Next, note that because $u$ is bounded from above and $v$ is bounded from below there cannot be a sequence $\left(t_{n}, x_{n}, y_{n}\right)_{n=1}^{\infty}$ in $[0, T) \times \bar{\Omega} \times \bar{\Omega}$ such that we would have

$$
\lim _{n \rightarrow \infty} \psi\left(t_{n}, x_{n}, y_{n}\right)=\sup _{t \in[0, T), x, y \in \bar{\Omega}} \psi(t, x, y)
$$

and

$$
\sup _{n \geq 1}\left(\left|x_{n}\right|+\left|y_{n}\right|+\frac{1}{T-t_{n}}\right)=\infty .
$$

It follows, because $u$ is upper and $v$ is lower semi-continuous, that there is a point $\left(t_{0}, x_{0}, y_{0}\right) \in[0, T) \times \bar{\Omega} \times \bar{\Omega}$ such that

$$
\psi\left(t_{0}, x_{0}, y_{0}\right) \geq \psi(t, x, y), \quad(t, x, y) \in[0, T) \times \bar{\Omega} \times \bar{\Omega} .
$$

On the other hand, we have by (77), (8), (15), (17), and the assumption that $\sigma>0$ that

$$
0 \leq \psi\left(t_{0}, x_{0}, y_{0}\right) \leq M_{0}-\frac{1}{2} \lambda\left|x_{0}-y_{0}\right|^{2}-\frac{1}{2} \epsilon\left|x_{0}\right|^{2},
$$

and it follows that

$$
\left|x_{0}-y_{0}\right| \leq \sqrt{\frac{2 M_{0}}{\lambda}}, \quad\left|x_{0}\right| \leq \sqrt{\frac{2 M_{0}}{\epsilon}} .
$$

Suppose that $t_{0}>0$ and that $x_{0}$ and $y_{0} \in \Omega$. Then we can apply [3, Thm. 8.3] to conclude (cf. the proof of [3, Thm. 8.2]) that there are numbers $a$ and $b$ and symmetric matrices $X$ and $Y$ such that

$$
\begin{aligned}
& \left(a, \lambda\left(x_{0}-y_{0}\right)+\epsilon x_{0}, X\right) \in \bar{P}_{\Omega}^{2,+}\left(u, t_{0}, x_{0}\right) \quad \text { and } \\
& \left(b, \lambda\left(x_{0}-y_{0}\right), Y\right) \in \bar{P}_{\Omega}^{2,-}\left(v, t_{0}, y_{0}\right)
\end{aligned}
$$


and such that

$$
a-b=\frac{(1-\delta) \sigma}{T}+\frac{\epsilon}{\left(T-t_{0}\right)^{2}}
$$

and

$$
\begin{array}{r}
-(3 \lambda+\epsilon)\left(\begin{array}{ll}
I & 0 \\
0 & I
\end{array}\right) \leq\left(\begin{array}{cc}
X & 0 \\
0 & -Y
\end{array}\right) \leq(3 \lambda+\epsilon) \\
\left(\begin{array}{cc}
I & -I \\
-I & I
\end{array}\right) \\
+\left(\begin{array}{cc}
\left(\frac{\epsilon^{2}}{\lambda}+2 \epsilon\right) I & 0 \\
0 & -\epsilon I
\end{array}\right) .
\end{array}
$$

We conclude by (20) and (21), after subtracting the two inequalities that one gets from the definitions of subsolutions and supersolutions, that

$$
\frac{(1-\delta) \sigma}{T}+\frac{\epsilon}{\left(T-t_{0}\right)^{2}}+F\left(u\left(t_{0}, x_{0}\right), \mathbf{p}+\epsilon x_{0}, X\right)-G\left(v\left(t_{0}, y_{0}\right), \mathbf{p}, Y\right) \leq 0
$$

where $\mathbf{p} \stackrel{\text { def }}{=} \lambda\left(x_{0}-y_{0}\right)$.

Let $\mathbf{p}_{F} \stackrel{\text { def }}{=} \mathbf{p}+\epsilon x_{0}, \alpha_{*}(\underline{\mathbf{q}})=a(|\underline{\mathbf{q}}|)+\alpha(\underline{\mathbf{q}})$, and $\beta_{*}(\underline{\mathbf{q}})=b(|\underline{\mathbf{q}}|)+\beta(\underline{\mathbf{q}})$. By (22) we have for an arbitrary $\mathbf{e} \in \mathbb{R}^{d}$,

$$
\begin{array}{r}
\alpha_{*}\left(\mathbf{p}_{F}\right)\langle\mathbf{e}, X \mathbf{e}\rangle-\beta_{*}(\mathbf{p})\langle\mathbf{e}, Y \mathbf{e}\rangle=\left\langle\left(\begin{array}{c}
\sqrt{\alpha_{*}\left(\mathbf{p}_{F}\right)} \mathbf{e} \\
\sqrt{\beta_{*}(\mathbf{p}) \mathbf{e}}
\end{array}\right),\left(\begin{array}{cc}
X & 0 \\
0 & -Y
\end{array}\right)\left(\begin{array}{c}
\sqrt{\alpha_{*}\left(\mathbf{p}_{F}\right)} \mathbf{e} \\
\sqrt{\beta_{*}(\mathbf{p}) \mathbf{e}}
\end{array}\right)\right\rangle \\
\leq(3 \lambda+\epsilon)\left(\sqrt{\alpha_{*}\left(\mathbf{p}_{F}\right)}-\sqrt{\beta_{*}(\mathbf{p})}\right)^{2}\langle\mathbf{e}, \mathbf{e}\rangle+3 \epsilon \alpha_{*}\left(\mathbf{p}_{F}\right)\langle\mathbf{e}, \mathbf{e}\rangle,
\end{array}
$$

where we used $\lambda \geq \epsilon$. By choosing $\mathbf{e}$ to be the vectors in an orthonormal basis, and then adding, one gets

$$
\begin{aligned}
\alpha_{*}\left(\mathbf{p}_{F}\right) \operatorname{tr}(X)-\beta_{*}(\mathbf{p}) \operatorname{tr}(Y) \leq(3 \lambda+\epsilon) d\left(\sqrt{\alpha_{*}\left(\mathbf{p}_{F}\right)}-\sqrt{\beta_{*}(\mathbf{p})}\right)^{2} & \\
& +3 d \epsilon \alpha_{*}\left(\mathbf{p}_{F}\right)
\end{aligned}
$$

Similarly, we also get from (22) that

$$
\begin{aligned}
& \frac{a^{\prime}\left(\left|\mathbf{p}_{F}\right|\right)}{\left|\mathbf{p}_{F}\right|}\left\langle\mathbf{p}_{F}, X \mathbf{p}_{F}\right\rangle-\frac{b^{\prime}(|\mathbf{p}|)}{|\mathbf{p}|}\langle\mathbf{p}, Y \mathbf{p}\rangle \\
& \leq\left\langle\left(\begin{array}{c}
\sqrt{\frac{a^{\prime}\left(\left|\mathbf{p}_{F}\right|\right)}{\left|\mathbf{p}_{F}\right|}} \mathbf{p}_{F} \\
\sqrt{\frac{b^{\prime}(|\mathbf{p}|)}{|\mathbf{p}|}} \mathbf{p}
\end{array}\right),\left(\begin{array}{cc}
X & 0 \\
0 & -Y
\end{array}\right)\left(\begin{array}{c}
\sqrt{\frac{a^{\prime}\left(\left|\mathbf{p}_{F}\right|\right)}{\left|\mathbf{p}_{F}\right|}} \mathbf{p}_{F} \\
\sqrt{\frac{\left.b^{\prime}|\mathbf{p}|\right)}{|\mathbf{p}|}} \mathbf{p}
\end{array}\right)\right\rangle \\
& \quad \leq(3 \lambda+\epsilon)\left|\sqrt{\frac{a^{\prime}\left(\left|\mathbf{p}_{F}\right|\right)}{\left|\mathbf{p}_{F}\right|}} \mathbf{p}_{F}-\sqrt{\frac{b^{\prime}(|\mathbf{p}|)}{|\mathbf{p}|}} \mathbf{p}^{2}+3 \epsilon a^{\prime}\left(\left|\mathbf{p}_{F}\right|\right)\right| \mathbf{p}_{F} \mid .
\end{aligned}
$$

From it also follows that

$$
X \leq Y+2 \epsilon I \quad \text { and } \quad\|X\| \leq 3 \lambda+4 \epsilon .
$$

When we use (19), (24), (25), and (26) together with the fact that $u\left(t_{0}, x_{0}\right) \geq$ $v\left(t_{0}, y_{0}\right)$ because $\sigma>0$, we get from (23) and from the monotonicity properties of 
$f$ and $g$ that

$$
\begin{aligned}
& \sigma \leq \frac{T}{1-\delta} \sup _{\left|\mathbf{q}_{F}\right| \leq \sqrt{2 \epsilon M_{0}}(r, \mathbf{q}, X) \in D_{\lambda+2 \epsilon}}\left(g(r, \mathbf{q}, X-2 \epsilon I)-f\left(r, \mathbf{q}+\mathbf{q}_{F}, X\right)\right. \\
& +(3 \lambda+\epsilon) d\left(\sqrt{a\left(\left|\mathbf{q}+\mathbf{q}_{F}\right|\right)+\alpha\left(\mathbf{q}+\mathbf{q}_{F}\right)}-\sqrt{b(|\mathbf{q}|)+\beta(\mathbf{q})}\right)^{2} \\
& +(3 \lambda+\epsilon)\left|\sqrt{\frac{a^{\prime}\left(\left|\mathbf{q}+\mathbf{q}_{F}\right|\right)}{\left|\mathbf{q}+\mathbf{q}_{F}\right|}}\left(\mathbf{q}+\mathbf{q}_{F}\right)-\sqrt{\frac{b^{\prime}(|\mathbf{q}|)}{|\mathbf{q}|}} \mathbf{q}\right|^{2} \\
& \left.+3 d \epsilon\left(a\left(\mathbf{q}+\mathbf{q}_{F}\right)+\alpha\left(\mathbf{q}+\mathbf{q}_{F}\right)\right)+3 \epsilon a^{\prime}\left(\left|\mathbf{q}+\mathbf{q}_{F}\right|\right)\left|\mathbf{q}+\mathbf{q}_{F}\right|\right)^{+}
\end{aligned}
$$

Suppose next that $t_{0}>0$ and $y_{0} \in \partial \Omega$. Then it follows from (7) and (11) because $v\left(t_{0}, y_{0}\right) \geq 0$ that

$$
u\left(t_{0}, x_{0}\right)-v\left(t_{0}, y_{0}\right) \leq \omega\left(\left|x_{0}-y_{0}\right|\right) .
$$

It is clear that we get the same inequality if $x_{0} \in \partial \Omega$. From (15), (16), (17), and (18) we now conclude that

$$
\sigma \leq \frac{1}{\delta}\left(\omega\left(\left|x_{0}-y_{0}\right|\right)-\frac{\lambda}{2}\left|x_{0}-y_{0}\right|^{2}\right) .
$$

Suppose finally that $t_{0}=0$. By (17) and (18) we must have

$$
\begin{array}{r}
\delta \sigma+E_{0} \leq u\left(0, x_{0}\right)-v\left(0, y_{0}\right)-\frac{\lambda}{2}\left|x_{0}-y_{0}\right|^{2} \leq u_{0}\left(x_{0}\right)-v_{0}\left(y_{0}\right)-\frac{\lambda}{2}\left|x_{0}-y_{0}\right|^{2} \\
\leq\left|u_{0}\left(x_{0}\right)-u_{0}\left(y_{0}\right)\right|+E_{0}-\frac{\lambda}{2}\left|x_{0}-y_{0}\right|^{2},
\end{array}
$$

and by (4) we have (28).

Now we have two upper bounds for $\sigma$, i.e., (27) and (28), depending on whether $t_{0}$ is positive or not and whether at least one of the points lies on the boundary. Thus we get an upper bound of the form $\max \left\{\frac{\mu}{1-\delta}, \frac{\eta}{\delta}\right\}$ and by choosing $\delta=\frac{\eta}{\mu+\eta}$ it becomes $\mu+\eta$. Hence we have (trivially in the case $\sigma \leq 0$ as well)

$$
\begin{aligned}
\sigma \leq & \sup _{x, y \in \bar{\Omega}}\left(\omega(|x-y|)-\frac{\lambda}{2}|x-y|^{2}\right) \\
+T & \sup _{\left|\mathbf{q}_{F}\right| \leq \sqrt{2 \epsilon M_{0}}}\left(\operatorname { s u p } _ { ( r , \mathbf { q } , X ) \in D _ { \lambda + 2 \epsilon } } \left(g(r, \mathbf{q}, X-2 \epsilon I)-f\left(r, \mathbf{q}+\mathbf{q}_{F}, X\right)\right.\right. \\
& +(3 \lambda+\epsilon) d\left(\sqrt{a\left(\left|\mathbf{q}+\mathbf{q}_{F}\right|\right)+\alpha\left(\mathbf{q}+\mathbf{q}_{F}\right)}-\sqrt{b(|\mathbf{q}|)+\beta(\mathbf{q})}\right)^{2} \\
& +(3 \lambda+\epsilon)\left|\sqrt{\frac{a^{\prime}\left(\left|\mathbf{q}+\mathbf{q}_{F}\right|\right)}{\left|\mathbf{q}+\mathbf{q}_{F}\right|}}\left(\mathbf{q}+\mathbf{q}_{F}\right)-\sqrt{\frac{b^{\prime}(|\mathbf{q}|)}{|\mathbf{q}|}} \mathbf{q}\right|^{2} \\
& \left.\left.+3 d \epsilon a\left(\mathbf{q}+\mathbf{q}_{F}\right)+3 d \epsilon \alpha\left(\mathbf{q}+\mathbf{q}_{F}\right)+3 \epsilon a^{\prime}\left(\left|\mathbf{q}+\mathbf{q}_{F}\right|\right)\left|\mathbf{q}+\mathbf{q}_{F}\right|\right)\right)^{+}
\end{aligned}
$$

If now $x \in \bar{\Omega}$ and $t \in[0, T)$ are arbitrary, then we have by the definition of $\sigma$ that

$$
u(t, x)-v(t, x) \leq E_{0}+\sigma+\frac{\epsilon}{T-t}+\epsilon|x|^{2} .
$$


If we now use (29) in this inequality and then let $\epsilon \downarrow 0$, we get for $x \in \bar{\Omega}$ and $t \in[0, T)$

$$
\begin{aligned}
u(t, x)-v(t, x) \leq \sup _{y \in \bar{\Omega}}\left(u_{0}(y)-v_{0}(y)\right)^{+}+\sup _{r \geq 0}\left(\omega(r)-\frac{\lambda}{2} r^{2}\right) \\
+T \sup _{(r, \mathbf{q}, X) \in D_{\lambda}}\left(g(r, \mathbf{q}, X)-f(r, \mathbf{q}, X)+3 \lambda\left(\sqrt{|\mathbf{q}| a^{\prime}(|\mathbf{q}|)}-\sqrt{|\mathbf{q}| b^{\prime}(|\mathbf{q}|)}\right)^{2}\right. \\
\left.+3 \lambda d(\sqrt{a(|\mathbf{q}|)+\alpha(\mathbf{q})}-\sqrt{b(|\mathbf{q}|)+\beta(\mathbf{q})})^{2}\right)^{+}
\end{aligned}
$$

since $f$ is lower semi-continuous, $g$ is upper semi-continuous and the functions $\alpha$, $\beta, a, b, \underline{r} a^{\prime}(\underline{r})$, and $\underline{r} b^{\prime}(\underline{r})$ are continuous.

If we have $f=\bar{g}, a=b, \alpha=\beta$ and $u_{0}(x) \leq v_{0}(x)$ for all $x \in \bar{\Omega}$, then we let $\lambda \rightarrow \infty$ in (30) and conclude that $u(t, x) \leq v(t, x)$ for all $t \geq 0$ and $x \in \bar{\Omega}$, that is, comparison holds. If $u$ and $v$ are solutions of (2) and (3), respectively, then we can interchange the role of $u$ and $v$ above and conclude that (5) holds.

Thus it remains to show that there are solutions of (21) and (3). Uniqueness will then follow from the fact that comparison holds. The argument is essentially the same as in [3, Sec. 4] (but see also [4) and we consider equation (2) only. Note that for this argument there is nothing to be gained by not writing the equation in the form

$$
u_{t}+F\left(u, D_{x} u, D_{x}^{2} u\right)=0 .
$$

First we observe that we have the following version of [3] Lemma 4.2]: Let $\mathcal{W}$ be a set of subsolutions of equation (31) in $(0, \infty) \times \Omega$. If $W(t, x)=\sup \{w(t, x) \mid w \in$ $\mathcal{W}\}$, then $W^{*}$ is a subsolution of $u_{t}+F\left(u, D_{x} u, D_{x}^{2} u\right)=0$ in $(0, \infty) \times \Omega$, where $W^{*}$ is the upper semi-continuous envelope of $W$. The proof is almost exactly the same as in [3] and the convexity of $\Omega$ is, of course, not needed here.

Suppose for the moment that $u_{0} \in \mathcal{C}^{2}(\bar{\Omega} ; \mathbb{R})$ with bounded second derivatives and let

$$
c \stackrel{\text { def }}{=} \sup \left\{|F(r, \mathbf{p}, X)| \mid r=u_{0}(x), \mathbf{p}=D_{x} u_{0}(x), X=D_{x}^{2} u_{0}(x), x \in \Omega\right\} .
$$

Then we easily see that $-c \underline{t}+u_{0}(\underline{x})$ is a subsolution and $c \underline{t}+u_{0}(\underline{x})$ is a supersolution of (31) on $(0, \infty) \times \Omega$. Above we showed that the maximum of two subsolutions is a subsolution so we know that $\max \left\{\breve{u}_{0}(\underline{x}),-c \underline{t}+u_{0}(\underline{x})\right\}$ is a subsolution of (B1). Similarly, $\min \left\{\widehat{u_{0}}(\underline{x}), c \underline{t}+u_{0}(\underline{x})\right\}$ is a supersolution.

For $t \geq 0$ and $x \in \bar{\Omega}$ let

$$
\begin{aligned}
u(t, x) \stackrel{\text { def }}{=} & \sup \{w(t, x) \mid w \text { is a subsolution of }(2) \text { and if } s \geq 0 \text { and } y \in \bar{\Omega}, \\
& \text { then } \left.\max \left\{\breve{u_{0}}(y),-c s+u_{0}(y)\right\} \leq w(s, y) \leq \min \left\{\widehat{u_{0}}(y), c t+u_{0}(y)\right\}\right\} .
\end{aligned}
$$

If we let $u^{*}$ be the upper semi-continuous envelope of $u$, then it follows by the result above that $u^{*}$ is a subsolution of (31) and by the definition of $u$ and by (11) (and the corresponding inequality for $\breve{u}_{0}$ ) we conclude that the initial and boundary conditions are satisfied. Since we can use almost the same argument as in [3. proof of Thm. 4.1] to show that $u_{*}$ (the lower semi-continuous envelope of $u$ ) is a supersolution, we conclude from the fact that the comparison principle holds that $u_{*}=u^{*}$ and we have found a solution. 
If $u_{0}$ is not twice continuously differentiable, then we can approximate it by such functions and use the estimate contained in (15) to show that these approximations converge uniformly, and therefore their limit is a viscosity solution; cf. [3, Sec. 6].

\section{REFERENCES}

1. B. Cockburn, G. Gripenberg, and S-O. Londen. Continuous dependence on the nonlinearity of viscosity solutions of parabolic equations. J. Differential Equations, 170:180-187, 2001. CMP 2001:08

2. M. G. Crandall. Viscosity solutions: a primer. In Viscosity solutions and applications (Montecatini Terme, 1995), volume 1660 of Lecture Notes in Math., pages 1-43. Springer, Berlin, 1997. MR 98g:35034

3. M.G. Crandall, H. Ishii, and P.L. Lions. User's guide to viscosity solutions of second order partial differential equations. Bull. Amer. Math. Soc., 27:1-67, 1992. MR 92j:35050

4. D. Nunziante. Existence and uniqueness of unbounded viscosity solutions of parabolic equations with discontinuous time-dependence. Nonlinear Anal., 18(11):1033-1062, 1992. MR 93f:35125

5. T. Rockafellar. Convex analysis. Princeton University Press, Princeton, N.J., 1970. MR 43:445

Institute of Mathematics, Helsinki University of Technology, P.O. Box 1100, Fin02015 HUT, Finland

E-mail address: gustaf.gripenberg@hut.fi 